\title{
Balancing efficacy and financial toxicity in the management of metastatic castration-sensitive prostate cancer
}

\author{
Srikala S. Sridhar, MD, MSc, FRCPC \\ Princess Margaret Cancer Centre, Toronto, ON, Canada
}

Cite as: Can Urol Assoc J 2019;13(12):404-5. htrp://dx.doi.org/10.5489/cuaj.6346

See related article on page 396

W ith the rising costs of cancer care worldwide, we now need to carefully consider not only the efficacy and tolerability of novel therapeutic strategies but also their value. Value is broadly defined as healthcare outcomes per dollar spent. 'Traditionally, qualityadjusted life years (QALYs) and incremental cost-effectiveness ratios (ICERs), although not perfect, have been used as surrogate endpoints for value. QALY measures health as a combination of the duration of life and the health-related quality of life, while ICER is calculated as the difference in the expected cost of two interventions, divided by the difference in the expected QALYs. ICERs are often compared to a threshold ICER, which is referred to as the willingness to pay for health gain. ${ }^{2}$ Interventions with an ICER below the threshold are funded, whereas those with ICERs above tend not to be. Although there is no firm threshold in Canada, commonly accepted willingness-to-pay thresholds range from \$50 000-100 000/QALY gained.

The treatment landscape for de novo metastatic castration-sensitive prostate cancer (mCSPC) has evolved rapidly over the last five years. Androgen-deprivation therapy (ADT) remains the backbone of treatment, but recent studies have shown that the addition of docetaxel or abiraterone to ADT significantly improves outcomes and is now considered the standard of care. In this issue of CUAJ, Beca and colleagues report on the cost-effectiveness, from a Canadian public payer perspective, of administering six cycles of docetaxel with ADT compared to administering ADT alone in patients with high-volume mCSPC. ${ }^{3}$ The authors should be commended for conducting this study, as cost-effectiveness evaluations are critically important and given varying healthcare systems, cannot easily be extrapolated from one country to another.

In their study, Beca and colleagues used both a Markov model and a partitioned survival model for high-volume metastatic prostate cancer with three health states: mCSPC, metastatic castration-resistant prostate cancer, and death. Survival data were obtained from the CHAARTED trial, which first reported outcomes for patients with high-volume disease. ${ }^{4}$ Using Ontario costs data and utilities from the literature, they reported that docetaxel + ADT cost an additional \$25 757 and produced an extra 1.06 QALYs, resulting in an ICER of \$24 226/QALY gained. Results from one-way sensitivity analysis across wide ranges of estimates and a range of scenarios, including an alternate model structure, produced ICERs below $\$ 35$ 000/QALY gained in all cases, which falls within the willingness-to-pay thresholds.

The investigators concluded that the addition of docetaxel to ADT in high-volume mCSPC was an economically attractive approach. Of note, similar and consistent findings have been reported by other studies from Brazil, the U.K., China, and the U.S. ${ }^{5-8}$ This is likely due to the fact that docetaxel is a generic chemotherapy drug, is administered for a limited number of six cycles (18 weeks), and only has a short-term impact on quality of life. However, the benefit in low-volume mCSPC remains controversial and many patients and physicians would prefer to avoid chemotherapy if possible.

An alternative to chemotherapy in MCSPC is abiraterone, which is an orally administered androgen receptor-axis targeted agent. In the Latitude and STAMPEDE studies, adding abiraterone to ADT improved overall survival by about 38\% vs. ADT alone. ${ }^{9,10}$ The improvement in survival seen with the addition of either abiraterone and docetaxel to ADT in mCSPC has been substantial and of similar magnitude. The cost-effectiveness, however, significantly favors docetaxel. In a study by Ramamurthy et al, using progression-free survival (PFS) QALYs, the ICER for docetaxel vs. ADT was \$46 519/QALY compared to \$705 323/QALY for abiraterone, which falls well beyond the willingness-to-pay threshold. ${ }^{11}$ However, generic formulations of abiraterone are expected to be available soon and this may improve its cost-effectiveness.

To date, there are already three other positive trials in the mCSPC setting, including ARCHES (enzalutamide), TITAN (apalutamide), and ENZAMET (enzalutamide). ${ }^{12-14}$ Assuming comparable efficacy and quality of life, drug pricing will 
play a key role in determining which drugs are ultimately adopted and which are not. While there are fixed costs related to managing cancer complications, drug costs are, to some extent, driven by profit and return on investment for development. This is often above and beyond the cost of manufacturing and distribution and this price could, therefore, be negotiated to improve cost-effectiveness. At the same time, if strategies like administering a lower-dose abiraterone with food or intermittent dosing of the androgen receptor pathway inhibitors demonstrate comparable efficacy to standard dosing, these strategies could be used as a way to reduce costs of treatment and improve global access to these expensive drugs.

There have been significant advances and new treatment options for the management of mCSPC, translating into improved overall outcomes. In order to determine the most efficacious and cost-effective treatment options, we need to think more about harmonizing eligibility and endpoint criteria in clinical trials to help inform the optimal treatment strategy and at the same time, wherever possible, incorporate high-quality cost-effectiveness analyses into trial design. As we look ahead, the development of predictive biomarkers may one day allow us to better tailor available treatments to patients most likely to respond, and this may prove to be the most cost-effective strategy of all.

Competing interests: Dr. Sridhar has been an advisory board member for Astellas, AstraZeneca, Bayer, Janssen, Merck, and Roche; and has participated in several clinical trials.

\section{References}

1. Porter ME. What is value in health care? N Eng J Med 2010;363:2477-81. https://doi.org/10.1056/ NEJMp 1011024

2. Incremental cost-effectiveness ratio (ICER) [online]. (2016). York; York Health Economics Consortium; 2016. Available at: https://yhec.co.uk/glossary/incremental-cost-effectiveness-ratio-icer/. Accessed Nov. 4, 2019

3. Beca J, Habeeb M, Chan KKW, et al. Cost effectiveness of docetaxel in high-volume hormone sensitive metastatic prostate cancer. Can Urol Assoc J 2019;13:396-403. http://dx.doi.org/10.5489/cuai.5889

4. Sweeney $\mathrm{CJ}$, Chen $\mathrm{YH}$, Carducci $M$, et al. Chemo-hormonal therapy in metastatic hormone-sensitive prostate cancer. N Engl J Med 2015;373:737-46. https://doi.org/10.1056/NEJMoal503747

5. Aguiar PN Jr, Tan PS, Simko S, et al. Cost-effectiveness analysis of abiraterone, docetaxel, or placebo plus androgen-deprivation therapy for hormone-sensitive advanced prostate cancer. Einstein (Sao Paulo) 2019;17: eGS4414. https://doi.org/10.31744/einstein_journal/2019GS4414

6. Woods BS, Sideris E, Sydes MR, et al. Addition of docetaxel to first-line long-term hormone therapy in prostate cancer (STAMPEDE): Modelling to estimate long-term survival, quality-adjusted survival, and cost-effectiveness. Eur Urol Oncol 2018;1:449-58.

7. Liu M, Qu S, Liu Y, et al. Comparative clinical effects and cost-effectiveness of maximum androgen blockade, docetaxel with androgen-deprivation therapy, and ADT alone for the treatment of mHSPC in China. J Comp Eff Res 2019;8:865-77. https://doi.org/10.2217/cer-2018-0133

8. Sathianathen NJ, Alarid-Escudero F, Kuntz KM, et al. Cost-effectiveness analysis of systemic therapy for metastatic hormone-sensitive prostate cancer. Euro Urol Ocol 2019. [Epub ahead of print]. https://doi.org/10.1016/i.euo.2019.01.004

9. James ND, de Bono JS, Spears MR, et al. Abiraterone for prostate cancer not previously treated with hormone therapy. N Engl J Med 2017;377:338-51. https://doi.org/10.1056/NEJMoal 702900

10. Fizazi K, Tran N, Fein L, et al. Abiraterone plus prednisone in metastatic, castration-sensitive prostate cancer. N Engl J Med 2017;377:352-60. hitps://doi.org/10.1056/NEJMoal704174

11. Ramamurthy C, Handorf EA, Correa AF, et al. Cost-effectiveness of abiraterone vs. docetaxel in the treatment of metastatic hormone-naive prostate cancer. Urol Oncol 2019;37:688-95. https://doi.org/10.1016/i.urolonc.2019.05.017

12. Armstrong AJ, Szmulewitz RZ, Petrylak DP, et al. ARCHES: A randomized, phase 3 study of androgendeprivation therapy with enzalutamide or placebo in men with metastatic hormone-sensitive postate cancer. J Clin Oncol 2019:JC01900799. [Epub ahead of print]. https://doi.org/10.1200/JC0.19.00799

13. Chi KN, Agarwal N, Biartell A, et al. Apalutamide for metastatic, castration-sensitive prostate cancer. $N$ Engl J Med 2019;381:13-24. https://doi.org/10.1056/NEJMoa1903307

14. Davis ID, Martin AJ, Stockler MR, et al. Enzalutamide with standard first-line therapy in metastatic prostate cancer. N Engl J Med 2019;381:121-31. https://doi.org/10.1056/NEJMoal903835

Correspondence: Dr. Srikala S. Sridhar, Princess Margaret Hospital, Toronto, ON, Canada; srikala.sridhar@uhn.ca 